CLINICAL STUDY

\title{
Low testosterone levels predict all-cause mortality and cardiovascular events in women: a prospective cohort study in German primary care patients
}

\author{
Caroline Sievers, Jens Klotsche ${ }^{1}$, Lars Pieper ${ }^{1}$, Harald J Schneider ${ }^{2}$, Winfried März ${ }^{3,4,5}$, Hans Ulrich Wittchen ${ }^{1}$, \\ Günter K Stalla and Christos Mantzoros ${ }^{6}$ \\ Department of Endocrinology, Max Planck Institute of Psychiatry, Kraepelinstrasse 2-10, 80804 Munich, Germany, ${ }^{1}$ Institute of Clinical Psychology and \\ Psychotherapy, Technische Universitaet Dresden, 01187 Dresden, Germany, ${ }^{2}$ Medizinische Klinik, Ludwig-Maximilian University, Innenstadt, 80336 \\ Munich, Germany, ${ }^{3}$ Synlab Medizinisches Versorgungszentrum Heidelberg GmbH, 69214 Eppelheim, Germany, ${ }^{4}$ Medical Faculty Mannheim, Mannheim \\ Institute of Public Health, Social and Preventive Medicine, University of Heidelberg, 69120 Heidelberg, Germany, ${ }^{5}$ Clinical Institute of Medical and \\ Chemical Laboratory Diagnostics, Medical University Graz, A-8036 Graz, Austria and ${ }^{6}$ Division of Endocrinology, Diabetes, and Metabolism, Department \\ of Medicine, Harvard Medical School and Harvard School of Public Health, Beth Israel Deaconess Medical Center, Boston, Massachusetts O2215, USA
}

(Correspondence should be addressed to C Sievers; Email: csievers@mpipsykl.mpg.de)

\begin{abstract}
Objective: Although associations between testosterone and cardiovascular (CV) morbidity in women have been proposed, no large prospective study has evaluated potential associations between testosterone and mortality in women. The objective was to determine whether baseline testosterone levels in women are associated with future overall or CV morbidity and mortality.

Design: Prospective cohort study with a 4.5-year follow-up period.

Methods: From a representative sample of German primary care practices, 2914 female patients between 18 and 75 years were analyzed for the main outcome measures: CV risk factors, CV diseases, and all-cause mortality.

Results: At baseline, the study population was aged $57.96 \pm 14.37$ years with a mean body mass index of $26.71 \pm 5.17 \mathrm{~kg} / \mathrm{m}^{2}$. No predictive value of total testosterone for incident CV risk factors or $\mathrm{CV}$ diseases was observed in logistic regressions. Patients with total testosterone levels in the lowest quintile Q1, however, had a higher risk to die of any cause or to develop a CV event within the follow-up period compared to patients in the collapsed quintiles Q2-Q5 in crude and adjusted Cox regression models (all-cause mortality: Q2-Q5 versus Q1: crude hazard ratios (HR) 0.49, 95\% confidence interval (CI) 0.33-0.74; adjusted HR 0.62, 95\% CI 0.42-0.939; CV events: Q2-Q5 versus Q1: crude HR 0.54, 95\% CI 0.38-0.77; adjusted HR 0.68, 95\% CI 0.48-0.97). Kaplan-Meier curves revealed similar data. Conclusions: Low baseline testosterone in women is associated with increased all-cause mortality and incident $\mathrm{CV}$ events independent of traditional risk factors.
\end{abstract}

European Journal of Endocrinology 163 699-708

\section{Introduction}

Men have a higher risk of cardiovascular (CV) morbidity and mortality than women, and it has been hypothesized that high testosterone levels in women may be a critical factor exerting detrimental effects on the CV system (1). This is supported by findings from cross-sectional and few longitudinal studies, in which positive associations between total and/or bioavailable testosterone and insulin, insulin resistance, overweight, glucose, hemostatic and inflammatory markers, carotid artery intimal-medial thickness, and adverse lipid profiles have been reported (2-7). Also subjects with higher testosterone have higher risk for disease entities such as the metabolic syndrome (MS), type 2 diabetes mellitus (T2DM), and coronary artery disease (CAD) in cross-sectional and case-control studies as well as a few longitudinal studies (8-14).

Other studies did not report any significant relationships or have suggested a positive effect of testosterone on $\mathrm{CV}$ diseases in women. Golden, for instance, reported associations with insulin resistance, but did not find any association of increasing quartiles of bioavailable testosterone with T2DM in 1973 postmenopausal women after adjusted analyses (5). Bernini et al. (15) reported that higher androgen concentrations in women are related to lower carotid wall thickness, a proxy of carotid artery atherosclerosis, independent of other CV risk factors, and the only published prospective study on endogenous sex hormones and CV disease and ischemic heart disease death in postmenopausal women, the Rancho Bernardo study, reports no 
predictive role of either high or low testosterone on these outcomes during 19 years of follow-up period (16).

In a recent study, Tivesten et al. (17) reported a nearly doubled risk of death (hazard ratios (HR) 1.96, 95\% confidence interval (CI) 1.46-2.62) in men with low testosterone (quartile 1 versus quartiles 2-4) (17). To our knowledge, our study is the largest prospective study investigating the relationship of testosterone with overall mortality in women.

\section{Subjects and methods}

\section{Study population}

This study was performed as a prospective cohort study within the DETECT cohort (Diabetes Cardiovascular Risk-Evaluation: Targets and Essential Data for Commitment of Treatment; http://www.detect-studie.de), a large-scale, cross-sectional study with a 4.5-year prospective-longitudinal component (55 518 unselected patients, $59 \%$ women and $41 \%$ men, of 3188 primary care physicians) as previously described. The DETECT study received the approval of the ethics committee of the Carl Gustav Carus Medical faculty at the Technical University of Dresden (AZ: EK149092003; Date: 16.09.2003), and all patients gave written informed consent. Methods and design were presented in detail elsewhere (18-20). Blood samples were taken from a random subset of these patients $(n=7519)$ in 2003 to investigate a wide range of laboratory markers. For the laboratory subsample, a comprehensive 4.5-year follow-up period was conducted in 2007/2008. Testosterone levels were available in a random subsample of 2914 women who were finally included in this analysis.

In general, DETECT study attrition could be kept low (93.9 and $93.1 \%$ primary response rates) due to several measures in place such as payments for complete documentation and a systematic patient tracking system to monitor critical outcomes such as hospitalization or mortality. Therefore, information bias due to patient dropout should be low, and misclassification of the hard outcomes such as death should be minimized.

\section{Assessment of CV risk factors, CV diseases, and covariates}

Clinical assessments were conducted using i) standardized patient questionnaires, and ii) standardized clinical interview and assessments of physicians with regard to the presence and severity of a wide range of illnesses as previously described (20).

All information was gathered at baseline and at follow-up periods (in 2003, 2004, and 2007/2008).
Physicians were asked to diagnose diabetes mellitus, hyperlipidemia, hypertension, CAD, and other diagnoses using standardized questionnaires as previously described (20). Current medication with a focus on CV treatments was recorded. A total of 23 medications could be marked, including one checkbox for further medication (20).

The behavioral variables such as smoking status (coded as current smoker, ex-smoker, and non-smoker as well as numbers of cigarettes per day), physical activity (coded as less or more than $2 \mathrm{~h} /$ week), and alcohol consumption (reported as numbers of drinks per week) were based on patients' interview assessments at baseline as previously described $(18,20,21)$.

Physicians were instructed to measure weight, height, blood pressure, and waist circumference (WC) according to a standardized protocol as previously described (21). Systolic (SBP) and diastolic blood pressure (DBP) was measured by indirect cuff sphygmomanometry after several minutes of rest in a sitting position. Use of an appropriate cuff size was advised. WC was measured with a tape measure midway between the lowest rib and the pelvis. The following anthropometric parameters were calculated: body mass index (BMI, weight in $\mathrm{kg}$ divided by the square of height in meters) and waist-to-height ratio (WHtR, WC divided by measured height in $\mathrm{cm}$ ) as previously described (18, 20-22).

According to the guidelines of the WHO (WHO, 2000 EK IV; EASO, 2002 EK IV) and the German guidelines of the Deutsche Adipositas Gesellschaft (DAG), Deutsche Diabetes Gesellschaft (DDG), Deutsche Gesellschaft für Ernährung (DGE), and Deutsche Gesellschaft für Ernährungsmedizin (DGEM), a WHtR of $>0.54$ was defined as abnormal, indicating visceral obesity.

MS was defined according to the International Diabetes Federation (IDF). For the IDF classification, criterion No. 1 and criteria Nos $2-5$ needed to be fulfilled for women: 1 , WC $\geq 80 \mathrm{~cm} ; 2$, triglycerides $>150 \mathrm{mg} / \mathrm{dl}$ or intake of lipid-reducing therapy; 3 , high-density lipoprotein cholesterol (HDL-C) $<50 \mathrm{mg} / \mathrm{dl}$ or intake of lipid-reducing therapy; 4 , SBP $\geq 130 \mathrm{mmHg}$ or $\mathrm{DBP} \geq 85 \mathrm{mmHg}$ or intake of hypertensive drugs; 5 , fasting glucose $\geq 100 \mathrm{mg} / \mathrm{dl}$ or type 2 diabetes or intake of oral antidiabetics (www.idf. org/metabolic_syndrome). T2DM was defined as fasting blood glucose level $\geq 126 \mathrm{mg} / \mathrm{dl}$ or non-fasting blood glucose $\geq 200 \mathrm{mg} / \mathrm{dl}$ or intake of oral antidiabetic drugs.

All measured variables were used as continuous and/ or dichotomized variable as appropriate in this study.

\section{Assessment of death}

Death was recorded by the primary care physicians. The causes of death were collected, based on the information of the physician and by death certificate if available. Dates were defined by the date of death on the physicians report. Any CV event was defined as 
either angina pectoris, myocardial infarction (MI), percutaneous transluminal coronary angioplasty, bypass, surgery, stroke, transient ischemic attack, or CV death.

\section{Laboratory variables}

Blood samples were collected between 0800 and $1000 \mathrm{~h}$ and shipped by courier at room temperature within $24 \mathrm{~h}$ to the central laboratory at the Medical University of Graz (Austria). On arrival in the central laboratory, the samples were centrifuged immediately, and within another $24 \mathrm{~h}$, serum and plasma were stored at $-20{ }^{\circ} \mathrm{C}$ until further processing. Testosterone was determined in serum with an electrochemiluminescence immunoassay (Elecsys Autoanalyzer 2010, Modular analytics, Roche Diagnostics). The intra- and inter-assay coefficients of variation (CV) were 2.7 and $5.6 \%$ respectively. The measuring range of the testosterone assay was between 0.02 and $15 \mathrm{ng} / \mathrm{ml}$ (eight patients had testosterone levels of $0.02 \mathrm{ng} / \mathrm{ml}$ : none of the subjects had testosterone levels below $0.02 \mathrm{ng} / \mathrm{ml}$ ).

Immunological methods are commonly used to measure testosterone $(13,14)$. Our assay has been calibrated against gas chromatography-mass spectrometry (GC-MS) by the producer showing a strong positive correlation ( $r=0.997$; documentation Roche). Additionally, we performed a comparison study for testosterone in women in our own laboratory. The correlation between a liquid chromatography-mass spectrometry (LC-MS technique) and the Elecsys Autoanalyzer 2010 was $r=0.83$ (Fig. 1).

IGF1 was determined with an automated chemiluminescence system (Nichols Institute Diagnostics, San Clemente, CA, USA). The maximal intra- and interassay $\mathrm{CV}$ were 5 and $7 \%$ respectively.

Clinical chemical parameters such as albumin as well as cholesterol and triglycerides were determined on a Roche Modular automatic analyzer. Lipoproteins (HDL-C, low-density lipoprotein cholesterol (LDL-C), and very low-density lipoprotein cholesterol (VLDL-C)) were determined electrophoretically on the HELENA

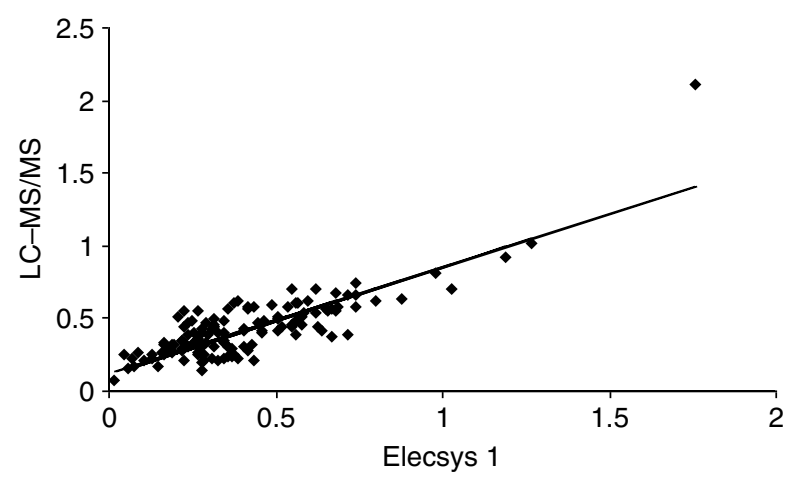

Figure 1 Correlation of Elecsys LC-MS/MS for testosterone in women in DETECT laboratory.
SAS-3/SAS-4 system. HbAlc was determined chromatographically on an ADAMS HA 8160 analyzing system (ARKRAY Inc., Kyoto, Japan). For all the parameters, reagents and secondary standards were used as recommended by the manufacturers. Inter-assay $\mathrm{CV}$ of these methods are provided elsewhere (20).

\section{Statistical analysis}

Women were stratified according to testosterone quintiles at baseline (testosterone quintiles: Q1, 0.020-0.237 ng/ml; Q2, 0.238-0.343 ng/ml; Q3, $0.344-0.472 \mathrm{ng} / \mathrm{ml}$; Q4, 0.473-0.655 ng/ml; Q5, 0.657-9.340 ng/ml). Quintiles were used for better discrimination given that increased $n$ value allowed us to do so. Further analyses were performed (i) contrasting Q2-Q5 against Q1. At baseline, $11.2 \%$ presented with elevated total testosterone levels (total testosterone $>0.82 \mathrm{ng} / \mathrm{ml}$ as defined by Roche Diagnostics) over all age groups. In premenopausal women 13.25\% and in postmenopausal women $9.4 \%$ of the patients fulfilled the criteria of hyperandrogenemia.

For descriptive statistics, we performed ANOVA in case of normally distributed continuous variables or $\chi^{2}$-tests in case of discrete outcomes to compare differences between quintiles (Table 1).

Spearman rank correlations were calculated for the univariate associations between testosterone and CV risk factors (data not shown).

Univariate and multivariate models were applied adjusting in different models for baseline age, BMI, smoking, IGF1 and albumin, menopausal status, physical activity, alcohol use, hypertension, hyperlipidemia, and diabetes mellitus as appropriate (Table 2).

Cox proportional hazards regression models were performed to analyze the relationship between testosterone and all-cause mortality and CV events (Tables 3 and 4). Unadjusted and adjusted (for age, BMI, and smoking) Kaplan-Meier survival curves contrast survival over time between testosterone quintiles Q2-Q5 versus Q1 (Fig. 2).

Data were analyzed using the statistical software SAS (Version 9.2, SAS Institute Inc., Cary, NC, USA). All $P$ values presented are two tailed; $P<0.05$ was considered statistically significant.

This manuscript was written in accordance with the STROBE statement, giving guidelines for reporting observational studies (23).

\section{Results}

At baseline, the age of the study population was $57.96 \pm 14.37$ (mean \pm s.D.) years, with a mean height of $163.6 \pm 6.50 \mathrm{~cm}$, a mean weight of 71.49 $\pm 14.22 \mathrm{~kg}$, and a mean BMI of $26.71 \pm 5.17 \mathrm{~kg} / \mathrm{m}^{2}$. Out of 2914 women, 1394 were postmenopausal and 1185 were premenopausal (with unknown status for the remaining 335 women). 
Table 1 Patients' characteristics in relation to testosterone quintiles (Q1-Q5) at baseline 2003. Values are expressed as geometric means or numbers. Reported $P$ values according to ANOVA or $\chi^{2}$-tests.

\begin{tabular}{|c|c|c|c|c|c|c|}
\hline & \multicolumn{5}{|c|}{ Testosterone quintiles } & \multirow[b]{2}{*}{$P$ value } \\
\hline & Q1 & Q2 & Q3 & Q4 & Q5 & \\
\hline \multicolumn{7}{|l|}{ Age } \\
\hline Age, years (mean) & 62.5 & 60.5 & 58.1 & 55.3 & 53.5 & $<0.001$ \\
\hline Postmenopausal women $(n)$ & 315 & 311 & 284 & 258 & 226 & $<0.001$ \\
\hline \multicolumn{7}{|l|}{ Anthropometric parameters } \\
\hline Weight, kg (mean) & 70.0 & 71.7 & 72.0 & 71.2 & 71.9 & NS \\
\hline Height, cm (mean) & 162.6 & 163.3 & 163.9 & 164.1 & 164.2 & 0.0003 \\
\hline Waist circumference, $\mathrm{cm}$ (mean) & 90.8 & 91.6 & 90.5 & 89.5 & 90.0 & NS \\
\hline Hip circumference, cm (mean) & 104.3 & 105.5 & 105.3 & 104.2 & 104.3 & NS \\
\hline WHR (mean) & 0.87 & 0.87 & 0.86 & 0.86 & 0.86 & NS \\
\hline WHtR (mean) & 0.56 & 0.56 & 0.55 & 0.55 & 0.55 & 0.025 \\
\hline $\mathrm{BMI}, \mathrm{kg} / \mathrm{m}^{2}$ (mean) & 26.7 & 27.2 & 26.9 & 26.6 & 26.7 & NS \\
\hline \multicolumn{7}{|l|}{ Risk factors } \\
\hline Systolic blood pressure, mmHg (mean) & 133.5 & 133.6 & 132.3 & 131.2 & 129.7 & 0.008 \\
\hline Diastolic blood pressure, $\mathrm{mmHg}$ (mean) & 79.4 & 79.7 & 80.1 & 78.8 & 79.2 & NS \\
\hline Mean blood pressure, $\mathrm{mmHg}$ (mean) & 105.9 & 106.0 & 105.9 & 104.6 & 104.2 & NS \\
\hline Total cholesterol, mg/dl (mean) & 230.7 & 228.7 & 231.7 & 222.3 & 230.7 & $<0.001$ \\
\hline LDL cholesterol, mg/dl (mean) & 129.6 & 128.3 & 131.4 & 124.5 & 126.0 & 0.009 \\
\hline HDL cholesterol, mg/dl (mean) & 60.7 & 60.6 & 60.8 & 60.4 & 57.9 & NS \\
\hline Triglycerides, $\mathrm{mg} / \mathrm{dl}$ (mean) & 144.9 & 139.8 & 133.7 & 125.9 & 128.8 & 0.002 \\
\hline Fasting plasma glucose, mg/dl (mean) & 96.2 & 100.9 & 101.0 & 98.8 & 101.0 & NS \\
\hline HbAlc, \% (mean) & 5.5 & 5.6 & 5.6 & 5.5 & 5.5 & 0.041 \\
\hline \multicolumn{7}{|l|}{ Biomarkers } \\
\hline Albumin, g/dl (mean) & 4.4 & 4.5 & 4.5 & 4.5 & 4.5 & $<0.001$ \\
\hline IGF1, ng/ml (mean) & 117.6 & 120.5 & 125.1 & 134.9 & 140.6 & $<0.001$ \\
\hline Testosterone, $\mathrm{ng} / \mathrm{ml}$ (mean) & 0.16 & 0.29 & 0.41 & 0.55 & 1.43 & $<0.001$ \\
\hline \multicolumn{7}{|l|}{ Behavioral variables } \\
\hline Physical activity, > 2 h/week $(n)$ & 273 & 292 & 283 & 276 & 270 & NS \\
\hline Current smoker $(n)$ & 39 & 52 & 71 & 92 & 93 & $<0.001$ \\
\hline Cigarets per day (current smokers) (mean) & 16.2 & 13.6 & 12.1 & 12.8 & 13.4 & 0.03 \\
\hline Current alcohol drinkers (regular/daily) $(n)$ & 13 & 20 & 25 & 24 & 13 & 0.04 \\
\hline Numbers of drinks per week (mean) & 2.06 & 2.09 & 2.03 & 2.35 & 2.26 & 0.01 \\
\hline
\end{tabular}

Table 1 presents the distribution of patients' characteristics with regard to testosterone levels in quintiles (Q1-Q5). Using ANOVA and $\chi^{2}$-tests for binary variables, we found that women with low testosterone levels were significantly older and more likely to be postmenopausal. Arithmetic means of anthropometric measures were not significantly different between the quintiles Q1-Q5 besides a higher WHtR in the lower testosterone quintiles.

Significant differences between testosterone quintiles were additionally found for SBP, total cholesterol, LDL-C, triglycerides, HbAlc, albumin, IGF1, as well as alcohol drinking and smoking status. Mean DBP, mean blood pressure, HDL-C, fasting glucose, and physical activity were not differently distributed between testosterone quintiles.

Spearman correlations between testosterone with $\mathrm{CV}$ risk factors revealed negative correlations of testosterone with age, menopausal status, waist-to-hip ratio (WHR), WHtR, SBP, mean blood pressure, total cholesterol, HDL-C, triglycerides, HbAlc, and smoking status as per cigarettes per day (data not shown). Significant positive correlations for total testosterone were found for height, albumin, IGF1, and alcohol consumption as per numbers of drinks per week (data not shown). As expected, IGF1 was highly correlated with many of the risk factors under investigation (18).

\section{Total testosterone in relation to $\mathrm{CV}$ risk factors and incident $C V$ diseases during the follow-up period}

In prospective analyses, we excluded patients with the respective prevalent $\mathrm{CV}$ risk factor or disease at baseline as appropriate. Subsequently, we calculated the incidences of $\mathrm{CV}$ risk factors (adverse anthropometric measures, hypertension, hyperlipidemia, T2DM, and MS) and CV diseases (CAD, MI, and stroke) during the follow-up period of 4.5 years.

Table 2 reports on the odds ratios (ORs) for newly diagnosed CV risk factors and diseases with respect to baseline testosterone levels stratified in quintiles. In unadjusted models, we observed lower incidences of hypertension in patients with testosterone levels in quintiles Q2, Q3, Q4, and Q5 or collapsed Q2-Q5 compared to $Q 1$ at baseline $(P$ for trend $=0.0370)$. The incidence of being overweight was higher in Q2 


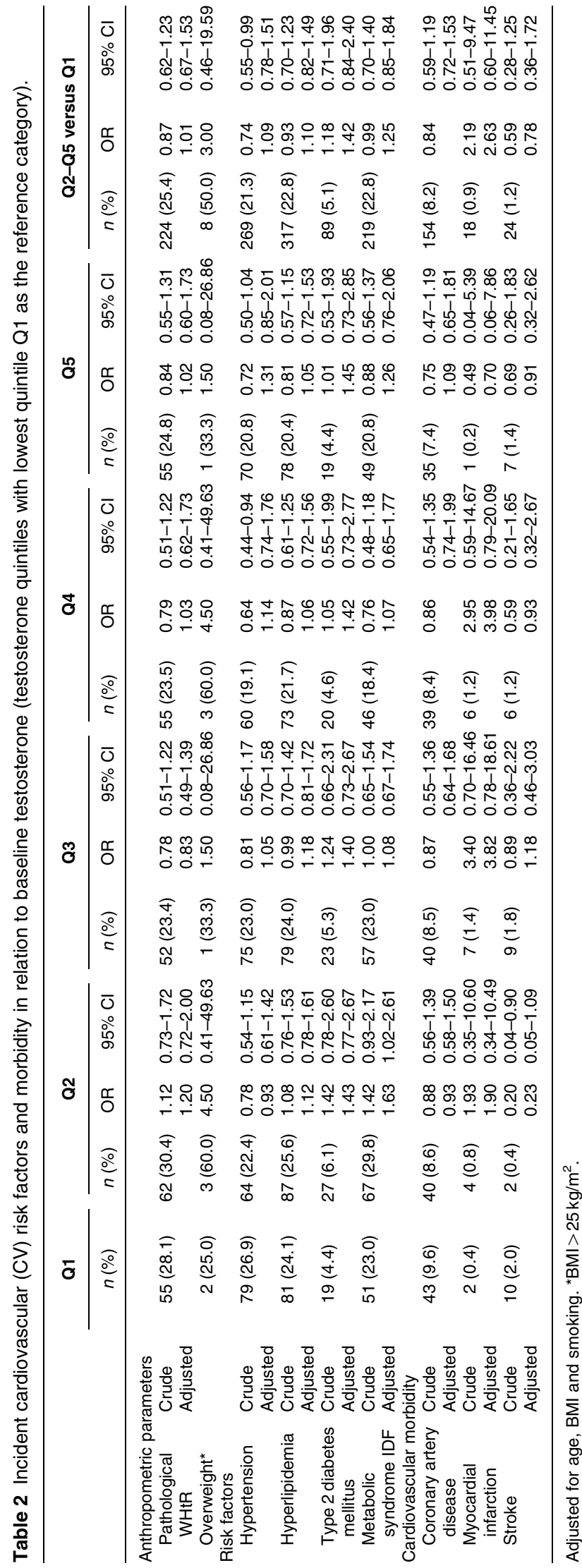

and Q4 compared to Q1 and in collapsed quintiles $Q 2-Q 5$ versus $Q 1$ ( $P$ for trend $=0.0055$ ). However, further adjustments abolished these effects indicating a strong confounding effect of age and BMI for hypertension.

Additional analyses adjusting for the presence of disease at baseline rather than exclusion of patients with prevalent diseases were largely confirmatory of the analyses presented herein.

When analyzing the predictive value of testosterone levels for incident CV morbidity (CAD, MI, and stroke), crude analyses and further adjustments (for age, BMI or smoking or age, BMI, smoking, physical activity, IGF1, and albumin and where applicable diabetes, hypertension, and hyperlipidemia) led to non-significant results of all three investigated outcomes (CAD, MI, and stroke) (Table 2). Additional analyses adjusting for baseline diseases rather than exclusion of patients with prevalent diseases were largely confirmatory of the analyses presented herein.

\section{Total testosterone in relation to all-cause mortality and CV events during the follow-up period (HR)}

To evaluate independent associations of total testosterone with all-cause mortality (Table 3 ) and any CV events (Table 4) before and after adjusting for age, BMI, and smoking, and for age, menopausal status, BMI, WHR, SBP, LDL-C, HDL-C, triglycerides, HbAlc, albumin, alcohol, and smoking, and for age, menopausal status, BMI, WHR, SBP, LDL-C, HDL-C, triglycerides, HbAlc, albumin, alcohol, smoking, and antihypertensive treatment, we performed Cox proportional hazard regressions.

Patients with testosterone levels in the lowest quintile Q1 (testosterone $<0.237 \mathrm{ng} / \mathrm{ml}$ ) had a higher risk of death from any cause within the follow-up period of 4.5 years compared with patients in Q3, Q4, or Q5 and the collapsed quintiles Q2-Q5 in crude and adjusted models (Q2-Q5 versus Q1: crude HR $0.49,95 \%$ CI $0.33-0.74$; adjusted HR 0.62, 95\% CI 0.42-0.939) (Table 3).

Similar results were found for $\mathrm{CV}$ events in crude and adjusted models (Q2-Q5 versus Q1: crude HR $0.54,95 \%$ CI $0.38-0.77$; adjusted HR $0.68,95 \%$ CI 0.48-0.97) (Table 4).

In sensitivity analyses, these results were also confirmed collapsing Q3-Q5 contrasted against Q1 or Q1+Q2 (data not shown).

We further investigated the importance of prevalent diseases on the association between testosterone and mortality as previously described (22). Therefore, we excluded women with prevalence of diabetes mellitus $(n=455), \mathrm{CV}$ diseases $(n=276)$, and cancer $(n=97)$ at baseline.

The adjusted HR for all-cause mortality and CV events remain significantly lower for patients in quintiles Q2-Q5 compared with women in Q1 if patients with diabetes mellitus ( $P=0.03$ and 0.016 respectively) 


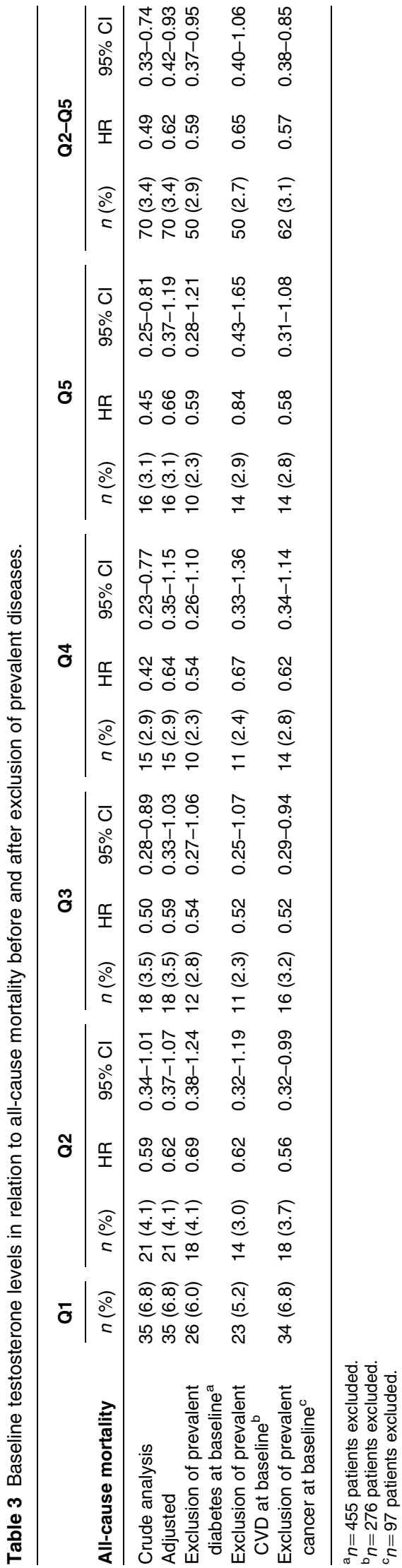

and cancer $(P=0.007$ and 0.038 respectively) were excluded (Tables 3 and 4). However, when CAD at baseline was excluded, significance for both all-cause mortality and CV events was reduced to a marginal level $(P=0.087$ and 0.076 respectively).

In additional sensitivity analyses, we excluded female patients on oral contraceptives or postmenopausal hormone therapy and hyperandrogenemia including interaction models with similar outcomes as described.

Figure 2 presents the cumulative survival curves for subjects in the testosterone lowest quintiles Q1 with higher all-cause mortality and CV events compared to female patients in quintiles Q2-Q5 (Fig. 2).

\section{Discussion}

In this large prospective cohort study, we investigated the predictive value of total testosterone on the incidence of $\mathrm{CV}$ risk factors, $\mathrm{CV}$ diseases, and mortality in female primary care patients in Germany.

The main finding of our study was the observation that all-cause mortality and $\mathrm{CV}$ events in female patients with low testosterone levels $(\mathrm{Q} 1$, testosterone $<0.237 \mathrm{ng} / \mathrm{ml}$ ) are elevated compared with patients with testosterone levels in quintiles Q2-Q5, and that this association is largely independent of traditional risk factors. However, no significant prospective associations were found in unadjusted and adjusted analyses in relation to new onset, incident, $\mathrm{CV}$ risk factors and $\mathrm{CV}$ diseases, implying that, if any, the main association of low testosterone could be not so much with disease occurrence but with disease progression, severity, and/or outcome. Our observational study, albeit prospective and incorporate the time sequence criterion for causality, cannot prove causality. Two hypotheses might explain our findings: i) low testosterone in women may cause or worsen disease and therefore leads to increased mortality rates or ii) low testosterone is simply a marker of disease or poor health.

The first hypothesis may be supported by prior clinical observations in men. Several epidemiological studies in men have demonstrated that low testosterone is associated with higher prevalence and incidence of CV risk factors, CV diseases, and mortality $(17,24,25)$. This phenomenon could be mediated through adverse direct and indirect effects of low testosterone on vascular tone, lipid and glucose metabolism, and the hemostatic system (25). In women, however, consequences of low testosterone or androgen deficiency on CV risk factors and diseases are poorly studied. Miller (26), for instance, reviewed that physiological testosterone replacement in women shows a positive effect on the restoration of a normal body composition, however, not consistently (27).

The second hypothesis is that low testosterone is a marker of poor health or more severe disease. This hypothesis is supported by pathological findings that 


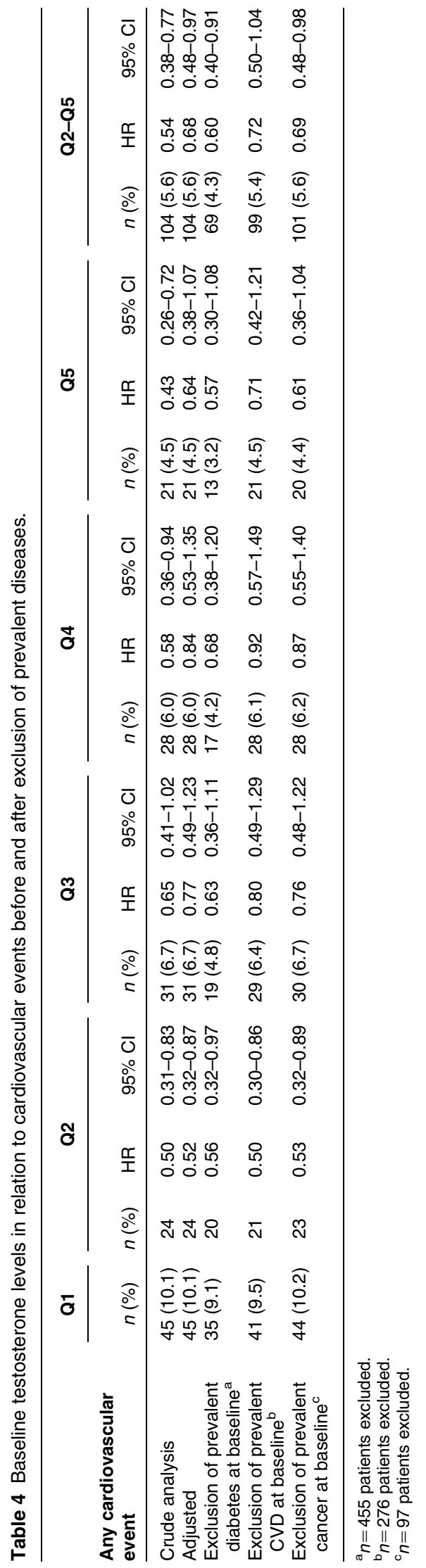

acute and chronic illness may decrease testosterone levels, which is, again, more comprehensively studied in men (28). It has been hypothesized that the stress following acute or chronic diseases with subsequent hypercortisolism may exert direct inhibitory effects on the hypothalamic-pituitary-gonadal system, resulting in hypogonadism. In women, it is known that a state of hypogonadotropic hypogonadism is not only associated with reduced estrogen, but also associated with lower testosterone levels (29).

Mohamad et al. (30), Dobrzycki et al. (31), Pugh et al. (32), and other authors reported that in men, specifically, CV diseases such as CAD are associated with hypoandrogenemia, but the results for women are less well studied and/or conclusive (30-34).

Another argument for the described hypothesis is supported by our results presented in Table 4. The exclusion of patients with prevalent $\mathrm{CV}$ diseases and prevalent CV disease or diabetes mellitus or cancer (but not prevalent diabetes mellitus alone or cancer alone) attenuated the statistical significance for all-cause mortality and CV events. We can therefore hypothesize that low testosterone is not only a marker of poor health, in general, but also more specifically and perhaps mainly of $\mathrm{CV}$ morbidity and mortality.

Other than stress, underlying diseases causing low testosterone levels in women are oophorectomy, adrenal insufficiency, supraphysiologic glucocorticoid administration, GnRH administration, anorexia nervosa, or AIDS wasting. Although the prevalence of patients with these diseases in our cohort is apparently extremely low, nevertheless they might have contributed to higher death rates due to the underlying disease independently of low testosterone levels.

To our knowledge, this is the most comprehensive study on this topic in a sample that includes both preand postmenopausal women. Only one smaller scale epidemiological study in 651 postmenopausal women investigated mortality over 19 years of follow-up period in relation to testosterone levels (16). The authors' comparisons of baseline total and bioavailable testosterone levels between female patients who died from CV disease and those who did not revealed lower baseline testosterone levels in the first group compared with the second group, which would be in line with our results. Differences with respect to significance of HR among this and our study could be due to differences in the two population samples (with both pre- and postmenopausal women included in our cohort with a lower mean age (57.9 versus 66.7 years)) and higher patient numbers $(n=2914$ versus $n=651)$ despite fewer years of follow-up period, which might have raised the power. Additionally, a very recent paper by Laughlin et al. (14) reported that low levels of testosterone are associated with an increased risk of coronary heart disease (CHD) events prospectively, which additionally underlines our findings (14). 

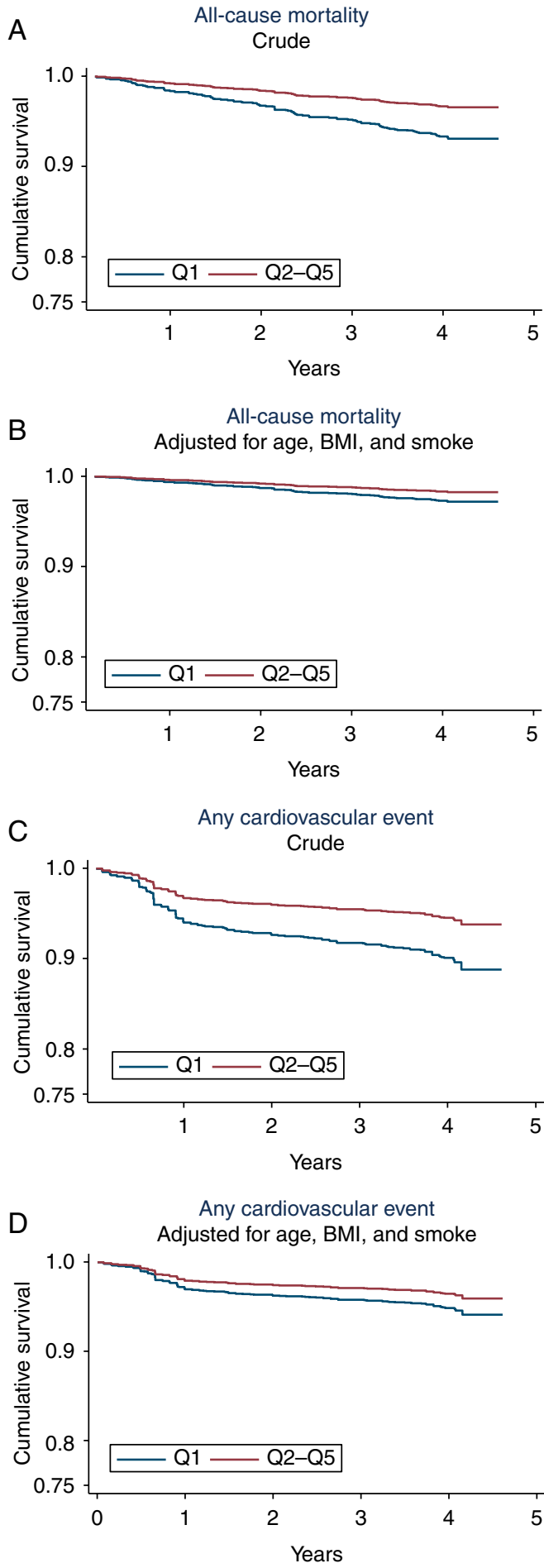

Figure 2 Unadjusted and adjusted (for age, BMI, and smoking) Kaplan-Meier curves for all-cause mortality ( $A$ and $B$ ) and any cardiovascular event $(C$ and $D)$. The graphs represent the cumulative survival of women in the lowest testosterone quintile Q1 compared to quintiles Q2-Q5.

\section{Strengths and limitations}

Testosterone measurements were performed using an electrochemiluminescence immunoassay (Elecsys system, Roche). This technique has been used as the standard to date and has been calibrated against the GC-MS technique showing a strong positive correlation. Ding et al. (8), Patel et al. (13), and Laughlin et al. (14) recently reported reliable results on testosterone in women with the immunoassay-based technique.

Our results are based on a single testosterone measurement. Despite circadian and other variability of testosterone levels, prior studies have demonstrated that intra-individual variance of total testosterone is small and similar in female patients with hyperandrogenemia or normal testosterone levels, and the authors propose that single androgen measurements are legitimate means to determine androgen status (35). Moreover, any misclassification would be random and would have suppressed effect estimates toward the null. Not taking the time of the menstrual cycle for premenopausal women into account might even further underestimate true relationships. We neither studied the role of free testosterone, SHBG, or estradiol nor their possible interactions with total testosterone. This is noteworthy, since independent associations of low SHBG, for instance, with the MS and CV risk factors in women with PCOS have been reported (36-38). On the other hand, previous studies found similar risks for total and free testosterone levels and CV risk factors in women.

To minimize measurement errors of the outcome and covariates, data checks for plausibility and completeness were implemented at data entry to further guarantee data quality. Potential confounders were addressed by adjustments for traditional clinical risk factors. Time differences occurred in the follow-up period between individual subjects and could have created nondifferential misclassification, which from the statistical point of view would have resulted in a suppression of effect estimates and loss of statistical significance. However, the fact that our results are significant indicates that our methods and statements should be valid.

To avoid confounding by those patients with extremely elevated testosterone levels, possibly associated with pathological conditions such as androgenproducing ovarian and adrenal neoplasms, Cushing's syndrome, polycystic ovary syndrome, and the intake of exogenous androgens, we repeated all analyses excluding those patients with pathological testosterone values above 0.82 or $>0.59 \mathrm{ng} / \mathrm{ml}$ (according to the Rotterdam criteria Rotterdam ESHRE/ASRM-Sponsored PCOS Consensus Workshop Group). In the second step, we excluded all patients on hormonal substitution. In addition to age, we also stratified and adjusted for menopausal status (including interaction models). Since we observed similar results, we decided to report on all women in DETECT. 
Although a stratified sampling scheme was used to recruit the patients in DETECT, the population under investigation reflects a primary care sample and the results may not be generalizable to the general German population.

Additionally, our study cohort consists of mainly relatively older women. The results and conclusions may therefore not be generalizable to a younger female population. Why we did not observe significant results for incident CV risk factors and diseases despite significant results for the Cox regression analysis on mortality needs to be discussed. We excluded respective prevalent risk factors and diseases in the logistic regression analyses (Table 2), which might have led to relatively low cases for the follow-up period and might have underpowered the study for these outcomes. On the other hand, it is conceivable that testosterone is rather a marker of progression to and/or indicator of serious disease and death than of the simple occurrence of $\mathrm{CV}$ diseases, which would be consistent with our second hypothesis as discussed above.

In conclusion, this study shows in a large primary care cohort that low baseline testosterone in relatively older women is associated with increased all-cause mortality and CV events, which is largely independent of traditional risk factors. Our results support the notion that the hormonal status of relatively older women might have consequences in relation to $\mathrm{CV}$ risk and the diagnostic work-up in women at risk for $\mathrm{CV}$ disease. Future studies are needed to confirm these results, to explore underlying mechanisms, and to determine whether this finding may prove to have direct implications on the clinical management of these patients.

\section{Declaration of interest}

There are no conflicts of interest for C Sievers, J Klotsche, L Pieper, H J Schneider, G K Stalla, and C Mantzoros. H U Wittchen and W März received an unrestricted educational grant for DETECT from Pfizer and additional fees for consulting.

\section{Funding}

The work was supported by a grant from the Deutsche Forschungsgemeinschaft (DFG) to support a program in clinical research techniques (SI-1425/1-1/2 to C Sievers). Diabetes Cardiovascular Risk-Evaluation: Targets and Essential Data for Commitment of Treatment (DETECT) is a cross-sectional and prospective, longitudinal, nationwide clinical epidemiological study. DETECT is supported by an unrestricted educational grant from Pfizer $\mathrm{GmbH}$, Karlsruhe, Germany.

\section{Acknowledgements}

Prof. Dr H U Wittchen is the principal investigator; L Pieper and J Klotsche are the staff members. Prof. H Lehnert (Lübeck), Prof. G K Stalla (Munich), and Prof. M A Zeiher (Frankfurt) are the members of the Steering Committee; Prof. W März (Graz), Prof. Dr S Silber (Munich), Prof. U Koch (Hamburg), Dr D Pittrow (Munich/Dresden), and Prof. M Wehling (Mannheim) are the members of the Advisory Board.

\section{References}

1 Rosano GM, Cornoldi A \& Fini M. Effects of androgens on the cardiovascular system. Journal of Endocrinological Investigation 200528 32-38.

2 Sutton-Tyrrell K, Wildman RP, Matthews KA, Chae C, Lasley BL, Brockwell S, Pasternak RC, Lloyd-Jones D, Sowers MF \& Torrens JI. Sex-hormone-binding globulin and the free androgen index are related to cardiovascular risk factors in multiethnic premenopausal and perimenopausal women enrolled in the Study of Women Across the Nation (SWAN). Circulation $20051111242-$ 1249. (doi:10.1161/01.CIR.0000157697.54255.CE)

3 Sowers MR, Jannausch M, Randolph JF, McConnell D, Little R, Lasley B, Pasternak R, Sutton-Tyrrell K \& Matthews KA. Androgens are associated with hemostatic and inflammatory factors among women at the mid-life. Journal of Clinical Endocrinology and Metabolism 200590 6064-6071. (doi:10. 1210/jc.2005-0765)

4 Haffner SM \& Valdez RA. Endogenous sex hormones: impact on lipids, lipoproteins, and insulin. American Journal of Medicine 1995 98 40S-47S. (doi:10.1016/S0002-9343(99)80058-8)

5 Golden SH, Dobs AS, Vaidya D, Szklo M, Gapstur S, Kopp P, Liu K \& Ouyang P. Endogenous sex hormones and glucose tolerance status in postmenopausal women. Journal of Clinical Endocrinology and Metabolism 200792 1289-1295. (doi:10.1210/jc.2006-1895)

6 Ouyang P, Vaidya D, Dobs A, Golden SH, Szklo M, Heckbert SR, Kopp P \& Gapstur SM. Sex hormone levels and subclinical atherosclerosis in postmenopausal women: the Multi-Ethnic Study of Atherosclerosis. Atherosclerosis 2009204 255-261. (doi:10. 1016/j.atherosclerosis.2008.08.037)

7 Wildman RP, Colvin AB, Powell LH, Matthews KA, EversonRose SA, Hollenberg S, Johnston JM \& Sutton-Tyrrell K. Associations of endogenous sex hormones with the vasculature in menopausal women: the Study of Women's Health Across the Nation (SWAN). Menopause 200815 414-421. (doi:10.1097/ gme.Ob013e318154b6f5)

8 Ding EL, Song Y, Manson JE, Rifai N, Buring JE \& Liu S. Plasma sex steroid hormones and risk of developing type 2 diabetes in women: a prospective study. Diabetologia 200750 2076-2084. (doi:10. 1007/s00125-007-0785-y)

9 Torrens JI, Sutton-Tyrrell K, Zhao X, Matthews K, Brockwell S, Sowers M \& Santoro N. Relative androgen excess during the menopausal transition predicts incident metabolic syndrome in midlife women: study of Women's Health Across the Nation. Menopause 200916 257-264. (doi:10.1097/gme.0b013e 318185e249)

10 Janssen I, Powell LH, Crawford S, Lasley B \& Sutton-Tyrrell K. Menopause and the metabolic syndrome: the Study of Women's Health Across the Nation. Archives of Internal Medicine 2008168 1568-1575. (doi:10.1001/archinte.168.14.1568)

11 Ahmed B, Bairey Merz CN, Johnson BD, Bittner V, Berga SL, Braunstein GD, Hodgson TK, Smith K, Shaw L, Kelsey SF \& Sopko G. Diabetes mellitus, hypothalamic hypoestrogenemia, and coronary artery disease in premenopausal women (from the National Heart, Lung, and Blood Institute sponsored WISE study). American Journal of Cardiology 2008102 150-154. (doi:10.1016/ j.amjcard.2008.03.029)

12 Rexrode KM, Manson JE, Lee IM, Ridker PM, Sluss PM, Cook NR \& Buring JE. Sex hormone levels and risk of cardiovascular events in postmenopausal women. Circulation $2003 \mathbf{1 0 8} 1688-1693$. (doi:10.1161/01.CIR.0000091114.36254.F3)

13 Patel SM, Ratcliffe SJ, Reilly MP, Weinstein R, Bhasin S, Blackman MR, Cauley JA, Sutton-Tyrrell K, Robbins J, Fried LP \& Cappola AR. Higher serum testosterone concentration in older women is associated with insulin resistance, metabolic syndrome, and cardiovascular disease. Journal of Clinical Endocrinology and Metabolism 2009 94 4776-4784. (doi:10.1210/jc.2009-0740)

14 Laughlin GA, Goodell V \& Barrett-Connor E. Extremes of endogenous testosterone are associated with increased risk of 
incident coronary events in older women. Journal of Clinical Endocrinology and Metabolism 200995 740-747. (doi:10.1210/ jc.2009-1693)

15 Bernini GP, Sgro M, Moretti A, Argenio GF, Barlascini CO, Cristofani R \& Salvetti A. Endogenous androgens and carotid intimal-medial thickness in women. Journal of Clinical Endocrinology and Metabolism 199984 2008-2012. (doi:10. 1210/jc.84.6.2008)

16 Barrett-Connor E \& Goodman-Gruen D. Prospective study of endogenous sex hormones and fatal cardiovascular disease in postmenopausal women. BMJ 1995311 1193-1196.

17 Tivesten A, Vandenput L, Labrie F, Karlsson MK, Ljunggren O, Mellstrom D \& Ohlsson C. Low serum testosterone and estradiol predict mortality in elderly men. Journal of Clinical Endocrinology and Metabolism 200994 2482-2488. (doi:10.1210/jc.20082650)

18 Schneider HJ, Klotsche J, Saller B, Bohler S, Sievers C, Pittrow D, Ruf G, Marz W, Erwa W, Zeiher AM, Silber S, Lehnert H, Wittchen HU \& Stalla GK. Associations of age-dependent IGF-I SDS with cardiovascular diseases and risk conditions: crosssectional study in 6773 primary care patients. European Journal of Endocrinology 2008158 153-161. (doi:10.1530/EJE-07-0600)

19 Schneider HJ, Sievers C, Klotsche J, Bohler S, Pittrow D, Lehnert H, Wittchen HU \& Stalla GK. Prevalence of low male testosterone levels in primary care in Germany: cross-sectional results from the DETECT study. Clinical Endocrinology 2009 70 446-454. (doi:10. 1111/j.1365-2265.2008.03370.x)

20 Wittchen HU, Glaesmer H, Marz W, Stalla G, Lehnert H, Zeiher AM, Silber S, Koch U, Bohler S, Pittrow D \& Ruf G. Cardiovascular risk factors in primary care: methods and baseline prevalence rates - the DETECT program. Current Medical Research and Opinion 200521 619-630. (doi:10.1185/0300 79905X38187)

21 Pittrow D, Pieper L, Klotsche J \& Wittchen H-U. In DETECT Ergebnisse einer klinisch-epidemiologischen Querschnitts- und Verlaufsstudie mit 55.000 Patienten in 3.000 Hausarztpraxen. Munich: Elsevier, Urban and Fischer GmbH, 2007.

22 Schneider HJ, Glaesmer $H$, Klotsche J, Bohler S, Lehnert H, Zeiher AM, Marz W, Pittrow D, Stalla GK \& Wittchen HU. Accuracy of anthropometric indicators of obesity to predict cardiovascular risk. Journal of Clinical Endocrinology and Metabolism 200792 589-594. (doi:10.1210/jc.2006-0254)

23 von Elm E, Altman DG, Egger M, Pocock SJ, Gotzsche PC \& Vandenbroucke JP. The Strengthening the Reporting of Observational Studies in Epidemiology (STROBE) statement: guidelines for reporting observational studies. Revista Española de Salud Pública $2008 \mathbf{8 2}$ 251-259. (doi:10.1590/S1135-57272 008000300002 )

24 Eckardstein A \& Wu FC. Testosterone and atherosclerosis. Growth Hormone and IGF Research 200313 (Supplement A) S72-S84. (doi:10.1016/S1096-6374(03)00059-5)

$25 \mathrm{Wu}$ FC \& von Eckardstein A. Androgens and coronary artery disease. Endocrine Reviews 200324 183-217. (doi:10.1210/er. 2001-0025)

26 Miller KK. Androgen deficiency: effects on body composition. Pituitary 200912 116-124. (doi:10.1007/s11102-008-0121-7)

27 Bhasin S, Calof OM, Storer TW, Lee ML, Mazer NA, Jasuja R, Montori VM, Gao W \& Dalton JT. Drug insight: testosterone and selective androgen receptor modulators as anabolic therapies for chronic illness and aging. Nature Clinical Practice. Endocrinology and Metabolism 20062 146-159. (doi:10.1038/ncpendmet0120)

28 Kaufman JM \& Vermeulen A. The decline of androgen levels in elderly men and its clinical and therapeutic implications. Endocrine Reviews 200526 833-876. (doi:10.1210/er.2004-0013)

29 Cappola AR, Ratcliffe SJ, Bhasin S, Blackman MR, Cauley J, Robbins J, Zmuda JM, Harris T \& Fried LP. Determinants of serum total and free testosterone levels in women over the age of 65 years. Journal of Clinical Endocrinology and Metabolism 200792 509-516. (doi:10.1210/jc.2006-1399)

30 Mohamad MJ, Mohammad MA, Karayyem M, Hairi A \& Hader AA. Serum levels of sex hormones in men with acute myocardial infarction. Neuro Endocrinology Letters $2007 \mathbf{2 8}$ $182-186$

31 Dobrzycki S, Serwatka W, Nadlewski S, Korecki J, Jackowski R, Paruk J, Ladny JR \& Hirnle T. An assessment of correlations between endogenous sex hormone levels and the extensiveness of coronary heart disease and the ejection fraction of the left ventricle in males. Journal of Medical Investigation 200350 162-169.

32 Pugh PJ, Channer KS, Parry H, Downes T \& Jone TH. Bio-available testosterone levels fall acutely following myocardial infarction in men: association with fibrinolytic factors. Endocrine Research 2002 28 161-173. (doi:10.1081/ERC-120015055)

33 Sablik Z, Samborska-Sablik A \& Goch JH. Concentrations of adrenal steroids and sex hormones in postmenopausal women suffering from coronary artery disease. Polski Merkuriusz Lekarski 200825 326-329.

34 Khatibi A, Agardh CD, Shakir YA, Nerbrand C, Nyberg P, Lidfeldt J \& Samsioe G. Could androgens protect middle-aged women from cardiovascular events? A population-based study of Swedish women: the Women's Health in the Lund Area (WHILA) Study Climacteric 200710 386-392. (doi:10.1080/13697130701377265)

35 Cho LW, Kilpatrick ES, Jayagopal V, Diver MJ \& Atkin SL. Biological variation of total testosterone, free androgen index and bioavailable testosterone in polycystic ovarian syndrome: implications for identifying hyperandrogenaemia. Clinical Endocrinology $2008 \mathbf{6 8}$ 390-394. (doi:10.1111/j.1365-2265.2007.03053.x)

36 Braunstein GD. Safety of testosterone treatment in postmenopausal women. Fertility and Sterility $2007 \mathbf{8 8}$ 1-17. (doi:10.1016/j.fertnstert.2007.01.118)

37 Braunstein GD, Johnson BD, Stanczyk FZ, Bittner V, Berga SL Shaw L, Hodgson TK, Paul-Labrador M, Azziz R \& Merz CN. Relations between endogenous androgens and estrogens in postmenopausal women with suspected ischemic heart disease. Journal of Clinical Endocrinology and Metabolism $2008 \mathbf{9 3}$ 4268-4275. (doi:10.1210/jc.2008-0792)

38 Ding EL, Song Y, Manson JE, Hunter DJ, Lee CC, Rifai N, Buring JE, Gaziano JM \& Liu S. Sex hormone-binding globulin and risk of type 2 diabetes in women and men. New England Journal of Medicine 2009361 1152-1163. (doi:10.1056/ NEJMoa0804381)

Received 28 June 2010

Accepted 4 August 2010 